\title{
The prognostic value of right ventricular deformation derived from cardiac magnetic resonance tissue tracking for all-cause mortality in light-chain amyloidosis patients
}

\author{
Hui Liu ${ }^{1 \#}$, Hang Fu ${ }^{1 \#}$, Ying-Kun Guo ${ }^{1}$, Zhi-Gang Yang ${ }^{2}$, Hua-Yan $\mathrm{Xu}^{1 \#}$, Xiao Shuai ${ }^{3}$, Rong Xu ${ }^{1}$, \\ Zhen-Lin $\mathrm{Li}^{2}$, Chun-Chao Xia ${ }^{2}$, Yong $\mathrm{He}^{4}$, Xiao-Yue Zhou \\ ${ }^{1}$ Department of Radiology, Key Laboratory of Obstetric \& Gynecologic and Pediatric Diseases and Birth Defects of Ministry of Education, West \\ China Second University Hospital, Sichuan University, Chengdu 610041, China; ${ }^{2}$ Department of Radiology, National Key Laboratory of Biotherapy, \\ ${ }^{3}$ Department of Hematology, ${ }^{4}$ Department of Cardiology, West China Hospital, Sichuan University, Chengdu 610041, China; ${ }^{5}$ MR Collaboration, \\ Siemens Healthcare Ltd., Shanghai 201318, China \\ Contributions: (I) Conception and design: H Liu, H Fu, YK Guo, ZG Yang; (II) Administrative support: YK Guo, ZG Yang; (III) Provision of \\ study materials or patients: Hy Xu, X Shuai, R Xu, Y He; (IV) Collection and assembly of data: ZL Li, CC Xia, XY Zhou; (V) Data analysis and \\ interpretation: H Liu, H Fu, YK Guo, ZG Yang; (VI) Manuscript writing: All authors; (VII) Final approval of manuscript: All authors. \\ "These authors contributed equally to this work. \\ Correspondence to: Ying-Kun Guo, MD, PhD. Department of Radiology, Key Laboratory of Obstetric \& Gynecologic and Pediatric Diseases and Birth \\ Defects of Ministry of Education, West China Second University Hospital, Sichuan University, 20\# South Renmin Road, Chengdu 610041, China. \\ Email: gykpanda@163.com; Zhi-Gang Yang, MD, PhD. Department of Radiology, West China Hospital of Sichuan University, Chengdu 610041, \\ China. Email: yangzg666@163.com.
}

Background: Early detection of right ventricular (RV) dysfunction is vital for determining the prognosis of light-chain amyloidosis (AL) patients. While few studies focused on RV deformation due to the limitation of research methods. The aim of this study was to determine the prognostic significance of RV myocardial strain in AL patients assessed by cardiac magnetic resonance (CMR) tissue tracking.

Methods: Sixty-four AL patients (28 females and 36 males, mean age $58 \pm 12.8$ years old; range $25-81$ years old) were enrolled from 1 October 2014 through 31 March 2017 and compared with 20 age- and sex-matched controls. Fifty-one AL patients met the criteria for cardiac amyloidosis (CA). Deformation parameters of both RV and left ventricle (LV) were measured by the CMR tissue tracking technique including myocardial global radial peak strain (GRPS), global circumferential peak strain (GCPS), and global longitudinal peak strain (GLPS). The follow-up time was 20 months or until the occurrence of death.

Results: Thirty-two (50\%) had preserved RV ejection fraction (RVEF $\geq 45 \%$ ). AL patients had significantly lower RV-GRPS $(20.3 \pm 2.12$ vs. $31.31 \pm 7.61)$, GCPS $(-2.12 \pm 0.88$ vs. $-13.71 \pm 2.53)$, and GLPS $(-5.33 \pm 0.64$ vs. $-14.239 \pm 2.99$ ) than controls even RVEF remain preserved (all $\mathrm{P}<0.001)$. Compared with controls and patients without CA, RV-GRPS $(12.26 \pm 1.26$ vs. $29.72 \pm 3.54, \mathrm{P}<0.001)$ and RV-GLPS $(-3.78 \pm 2.25$ vs. $-5.66 \pm 2.08, \mathrm{P}<0.05)$ were significantly lower in patients with $\mathrm{CA}$. Cox multivariate analyses demonstrated that RV-GRPS [hazard ratio $(\mathrm{HR})=0.93,95 \% \mathrm{CI}: 0.88-0.98, \mathrm{P}=0.007$ ] and Mayo stage were $(\mathrm{HR}=3.11$, 95\% CI: $1.30-7.41, \mathrm{P}=0.01)$ predictors of mortality in AL patients.

Conclusions: CMR tissue tracking is a feasible and highly reproducible technique for the analysis of RV deformation and could aid in the early diagnosis of RV involvement in AL patients. RV-GRPS of RV strain and Mayo stage provides prognostic information about mortality in AL patients.

Keywords: Cardiac magnetic resonance (CMR); tissue tracking; right ventricular (RV); cardiac amyloidosis (CA)

Submitted Oct 17, 2019. Accepted for publication Dec 17, 2019.

doi: $10.21037 / \mathrm{cdt} .2020 .01 .03$

View this article at: http://dx.doi.org/10.21037/cdt.2020.01.03 


\section{Introduction}

Immunoglobulin light-chain amyloidosis (AL) is the most common of all systemic amyloidosis subtypes, accounting for approximately $70 \%$ of all amyloidosis cases (1). Cardiac amyloidosis (CA) occurs in up to $80 \%$ of systemic $\mathrm{AL}$ patients and therefore represents one the most important clinical manifestations (1,2). Amyloid deposition in the heart typically presents as restrictive cardiomyopathy and often occurs with disproportionate signs of right ventricular (RV) failure (3). Clinically, RV function has been shown to have prognostic value in a variety of congenital abnormalities, pulmonary hypertension and dilated cardiomyopathy (4-8). Therefore, early detection of RV dysfunction is vital for determining the prognosis of AL patients.

To determine RV dysfunction early, 3D speckletracking echocardiography and cardiac magnetic resonance (CMR) tissue tracking have been regarded as early and sensitive makers for assessing subclinical deformations of both left ventricular (LV) and RV (9-15). However, CMR imaging is considered the gold standard technique for RV evaluation $(16,17)$ and could be used to detect subclinical changes in myocardial mechanics early in patients with RV deformation (18) due to the low spatial resolution and difficult acoustic windows $(19,20)$ of echocardiography. To the best of our knowledge, a few studies have evaluated $\mathrm{LV}$ dysfunction using CMR tissue tracking or tagging techniques in patients with AL $(21,22)$, and RV deformation assessment with CMR tissue tracking has not yet been reported in the literature. Thus, the aims of this study were (I) to assess and compare RV deformation in AL patients, especially those with preserved RV ejection fraction, and (II) further identify the most potent prognostic index of RV myocardial strain for all-cause mortality in AL patients.

\section{Methods}

\section{Study population}

This study was approved by our institutional review board. We retrospectively considered 71 consecutive AL patients (34 females and 37 males, mean age $59 \pm 10.2$ years old, range 25-81 years old) who had CMR examinations from 1 October 2014 through 31 March 2017. As Jaccard et al. (23) previously described, the diagnosis of amyloidosis was made based on a biopsy of subcutaneous fat or an involved organ with the demonstration of typical Conge red birefringence under polarized light. Amyloidosis was further confirmed by the detection of a monoclonal protein in the serum or urine and/or a monoclonal population of plasma cells in the bone marrow. The exclusion criteria were as follows: (I) patients with congenital heart disease, primary cardiomyopathy such as hypertrophic cardiomyopathy or coronary artery disease $(\mathrm{n}=1)$; (II) patients with poor quality CMR images $(\mathrm{n}=2)$; (III) patients with arrhythmia $(\mathrm{n}=2)$; and (IV) patients with pulmonary hypertension $(n=2)$. Finally, the study group consisted of 64 patients ( 28 females and 36 males, mean age $58 \pm 12.8$ years old, range $25-81$ years old). Patients selection flow diagram shows in Figure 1. According to A Consensus Opinion From the 10th International Symposium on Amyloid and Amyloidosis, CA was defined as an LV thickness $>12 \mathrm{~mm}$ without another known cause, as shown by echocardiography (24). A total of twenty age- and sexmatched healthy controls ( 8 females and 12 males, mean age $54 \pm 12.1$ years old, range $24-75$ years old) were enrolled as the normal control group. None of the included individuals had a history of heart diseases, systemic hypertension, or diabetes.

\section{Biomarkers, ECG and echocardiography}

In patients with AL amyloidosis, blood was collected within a week of CMR examination for the measurement of $\mathrm{N}$-terminal pro-brain natriuretic peptide (NTproBNP) and cardiac troponin T. Standard 12-lead resting electrocardiography (ECG) was performed in all patients prior to the CMR examination. All patients included in the study were examined by standard echocardiography before undergoing CMR focused on LV parameters.

\section{Standard CMR imaging protocol}

The CMR was performed with a 3.0-T scanner (Magnetom Tim Trio; Siemens Healthineers, Erlangen, Germany) using an eight-channel phased-array body coil during breath holding. Steady-state, free-precession, cine images were obtained along a consecutive short-axis covering the $\mathrm{LV}$ and $\mathrm{RV}$ from the mitral valve level to the apex and included 8 slices (TR/TE $37.66 \mathrm{~ms} / 1.2 \mathrm{~ms}$, flip angle $39^{\circ}$, FOV $280 \mathrm{~mm} \times 373 \mathrm{~mm}$, matrix size $146 \mathrm{~mm} \times 280 \mathrm{~mm}$, slice thickness $8 \mathrm{~mm}$ with no gap) as well as in the long axis (with two- and four-chamber views). Gadoliniumenhanced images were acquired in the same sections as the cine images $10-15$ minutes after intravenous injection of gadopentetate dimeglumine (dose: $0.1 \mathrm{~mL} / \mathrm{kg}$ body weight, flow rate: $2.5-3.0 \mathrm{~mL} / \mathrm{s}$, MultiHance $0.5 \mathrm{mmol} / \mathrm{mL}$; Bracco, Milan, Italy) using an inversion recovery TrueFISP 


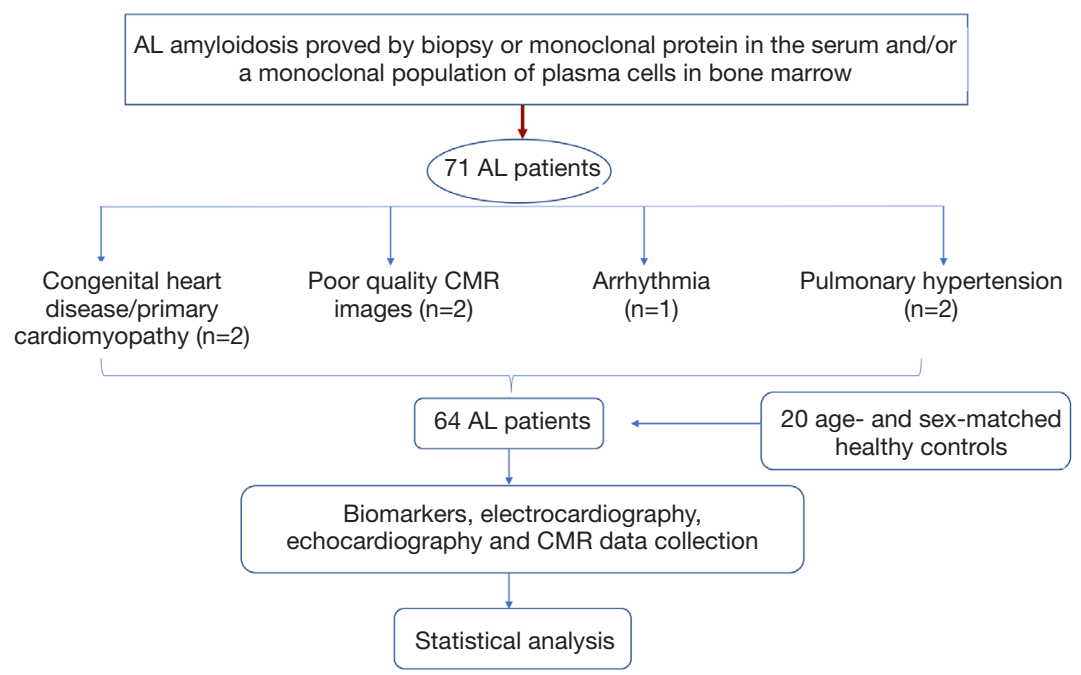

Figure 1 Patients selection flow diagram. Seventy-one AL patients underwent CMR were enrolled. Two patients with coronary artery disease, two patients with poor quality CMR images, one patient with arrhythmia and two patients with pulmonary hypertension were excluded. In brief, the study group consisted of sixty-four patients and twenty healthy controls.

sequence (TR/TE700/1.31 ms, flip angle $20^{\circ}$, FOV $320 \mathrm{~mm} \times 270 \mathrm{~mm}$, and slice thickness $8 \mathrm{~mm}$ ).

\section{Imaging analysis}

CMR studies were analysed offline by an experienced observer blinded to all patients clinical data using customized software (cvi42; version5.9.3; Circle Cardiovascular Imaging, Inc., Calgary, Canada). The endocardial and epicardial traces were performed manually in serial short-axis slices at the end-diastolic and endsystolic phases, and the papillary muscles and moderator bands were carefully excluded from the analysis. Global $\mathrm{LV} / \mathrm{RV}$ systolic function, including the LV/RV enddiastolic volume index (EDVi), end-systolic volume index (ESVi), and LV/RV ejection fraction (EF), were computed. Subsequently, LV myocardial strain analysis was performed by loaded the long-axis two-chamber, four-chamber and short-axis slices into the tissue tracking module. The analysis of RV myocardial strain was performed by loading the images of four-chamber and short-axis slices into the $\mathrm{RV}$ tissue tracking module. In all series, the endocardial and epicardial contours were delineated manually per slice at end-diastole. The global myocardial strain parameters were acquired automatically, including the global radial peak strain (GRPS), global circumferential peak strain (GCPS), and global longitudinal peak strain (GLPS) (Figure 2).
Positive and negative symbols represent direction. We defined peak strain as the absolute maximum strain over the entire cardiac cycle. The diagnosis of myocardium late gadolinium enhancement (LGE) was obtained by visual assessment, which required viewing an elevated signal in the myocardium within two orthogonal views.

Patients were divided into subgroups according to (I) preserved RVEF (RVEF\% $\geq 45 \%$ ) or reduced RVEF (RVEF\% < 45\%) (25), (II) patients with CA or without CA, and (III) CA patients with or without RV LGE.

\section{Clinical follow up}

The primary outcome was all-cause mortality after a review of hospital records or via a telephone call to the patient or their families. The follow-up was closed on 31 November 2018. The follow-up time was 20 months or until the occurrence of events. The time-to-event was defined as the time between CMR imaging and death.

\section{Reproducibility analysis}

To verify the reproducibility and reliability of RV functional assessment by CMR in our cohort, intra- and interobserver variability were calculated for RV strain parameter measurements in 26 randomly selected patients. For intraobserver variability, parameters were measured twice by 


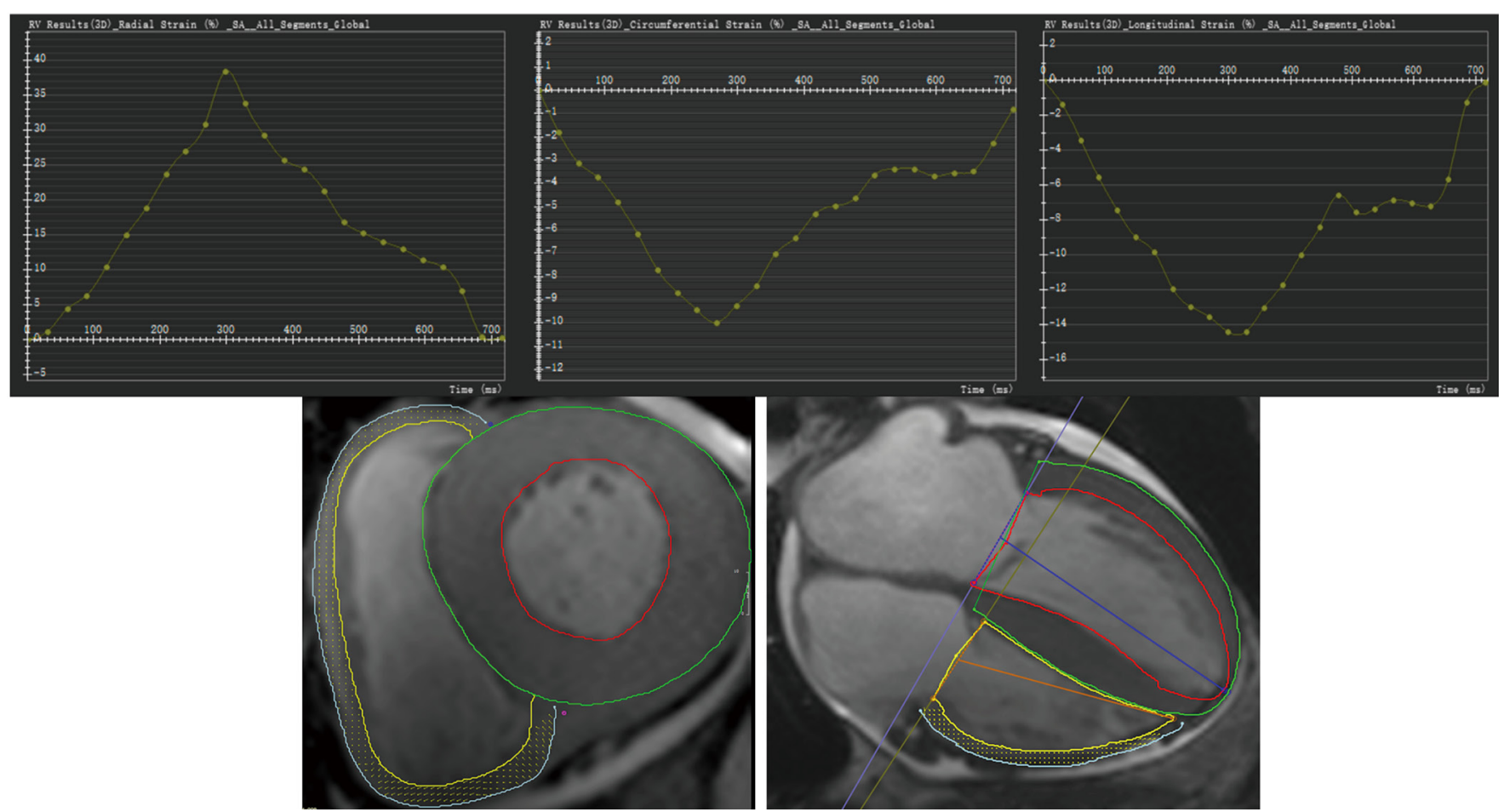

Figure 2 RV global radial peak strain, circumferential peak strain and longitudinal peak strain. The curve graphs show the global strain changes over time in a cardiac cycle in three directions of the right ventricle [abscissa represents time (ms), ordinate represents peak strain (\%)]. The short axis and four chambers of heart show the delineation of the inner and outer membranes. The yellow dots represent the myocardial voxel points. RV, right ventricular.

the same observer with a minimum interval of two weeks between serial assessments. Inter-observer variability was assessed using measurements obtained by a second independent observer blinded to all other analyses. These values are presented as means \pm standard deviation $(\mathrm{SD})$.

\section{Statistics analysis}

All statistical analyses were performed using a commercially available software package (SPSS for Windows, version 25.0; SPSS Inc, Chicago, IL; GraphPad version 7.00; San Diego, CA; and MedCalc, Version 9.3.0.0. MedCalc Software; Mariakerke, Belgium). All data were evaluated for normal distribution using the Kolmogorov-Smirnov test. Homogeneity of variance was evaluated using Levene's test. All results are expressed as the mean $\pm \mathrm{SD}$ or median [interquartile range (IQR), 25-75\%]. Independent samples $t$-tests and Mann-Whitney $\mathrm{U}$ tests were used to evaluate CMR parameters and the baseline characteristics of the normal and AL subgroups. Group percentages were compared using the Chi-square test or Fisher's exact test, where appropriate. Pearson correlation analysis was performed to examine the correlation between RV and LV global peak strain. Intra- and inter-observer variability were assessed using the intraclass correlation coefficient (ICC) method, and the 26 patient results are presented as Cronbach's $\alpha$ and $95 \%$ limits of agreement. Univariate and multivariate analyses were used to predict all-cause mortality in AL patients. NT-proBNP and troponin T were $\log (\ln )$-transformed to achieve normality for further analysis. Patients were divided into two groups by the median RV-GRPS. Kaplan-Meier survival curves were constructed to estimate the cumulative event rate for each end point according to the high or low RV-GRPS subgroup. The log-rank test was used to compare KaplanMeier survival curves. Statistical significance was considered a 2-tailed $\mathrm{P}<0.05$.

\section{Results}

\section{Baseline characteristics}

Baseline characteristics, biomarkers, ECG results and 
echocardiography parameters were recorded and are summarized in Table 1. Among the 64 AL patients, 1 had hypertension, 2 had diabetes, and 10 had a low-voltage by electrocardiography. Echocardiography examination found that tricuspid regurgitation and mitral regurgitation were observed in 9 and 12 patients, respectively. Laboratory tests showed that serum NT-proBNP and troponin T concentrations were 5,815.1 $(2,090.5-13,667.1) \mathrm{pg} / \mathrm{mL}$ and 83.5 (43.9-219.4) $\mu \mathrm{g} / \mathrm{L}$, respectively. There were 9 patients took Beta-blockers and 4 took cardiac glycosides. Thirtynine patients received chemotherapy and only four patients received stem cell transplantation.

\section{Subclinical RV myocardial deformation analysis}

Table 1 shows the characteristics of myocardial function and strain in both RV and LV in AL patients. To analyse the value of the index of $R V$ strain in the early diagnosis of $R V$ deformation in $\mathrm{AL}$ patients, we divided the $\mathrm{AL}$ patients into a preserved RVEF group ( $\mathrm{n}=32)$ and a reduced RVEF group $(\mathrm{n}=32)$. Similar to the results obtained for RVEF, LVEF was comparable between patients with preserved RVEF and healthy controls $(65.42 \pm 6.36$ vs. $60.68 \pm 12.63, \mathrm{P}=0.25)$. The LV-EDVi $(69.3 \pm 20.6$ vs. $42.2 \pm 8.3, \mathrm{P}=0.01)$ and $\mathrm{LV}-\mathrm{ESVi}$ $(27.1 \pm 14.2$ vs. $14.4 \pm 2.9, \mathrm{P}=0.21)$ also remined within the normal range in the preserved RVEF group.

For the strain analysis, RV-GRPS, RV-GLPS, and RVGCPS were significantly lower in AL patients than in controls $(\mathrm{P}<0.001$ for all comparisons). Most importantly, RV-GRPS $(8.5 \pm 1.04$ vs. $20.3 \pm 2.12, \mathrm{P}<0.001)$ and RV-GLPS $(-2.7 \pm 1.17$ vs. $-5.33 \pm 0.64, \mathrm{P}=0.03)$ were lower in patients with reduced RVEF than in those with preserved RVEF $(\mathrm{P}=0.02)$. As shown in Table 1, there was no significant difference in RV-GCPS between patients with preserved or reduced RVEF $(1.06 \pm 1.63$ vs. $-2.12 \pm 0.88, \mathrm{P}=0.15)$. In addition, a Pearson's correlation test indicated that there was a good correlation between RV-GRPS and LV-GRPS $(\mathrm{r}=0.56, \mathrm{P}=0.001)$ and a moderate correlation between $\mathrm{RV}$ GLPS and RV-LVGLPS ( $\mathrm{r}=0.32, \mathrm{P}=0.015)$ (Figure 3).

\section{$R V$ Myocardial deformation in $C A$}

As shown in Table 2, 51 (80\%) patients met the criteria for CA. RVEF was significantly lower in CA patients than in AL patients without $\mathrm{CA}(41.5 \pm 17.34$ vs. $53 \pm 14.34, \mathrm{P}<0.001)$. Similar decreases were observed in RV-GRPS $(12.26 \pm 1.26$ vs. $29.72 \pm 3.54, \mathrm{P}<0.001)$ and RV-GLPS $(-3.78 \pm 2.25$ vs. $-5.66 \pm 2.08, \mathrm{P}<0.05)$ between CA patients and those without CA. Similar to Table 1, no significant differences were found in RV-GCPS $(-2.41 \pm 1.72$ vs. $-0.78 \pm 0.75, \mathrm{P}>0.05)$ between AL patients with and without CA. Additionally, we found that $29(57 \%)$ of the 51 CA patients had RV LGE. Compared with AL patients without RV LGE, those with RV LGE had lower RVEF $(31.72 \pm 15.65 v s$. $45.48 \pm 12.70$, $\mathrm{P}=0.001)$ and $\mathrm{RV}-\mathrm{GRPS}(7.01 \pm 4.32$ vs. $13.75 \pm 9.59, \mathrm{P}=0.009)$ (Table 3).

Table 1 Baseline characteristics, cardiac function and myocardial strain in patients with preserved and reduced RVEF

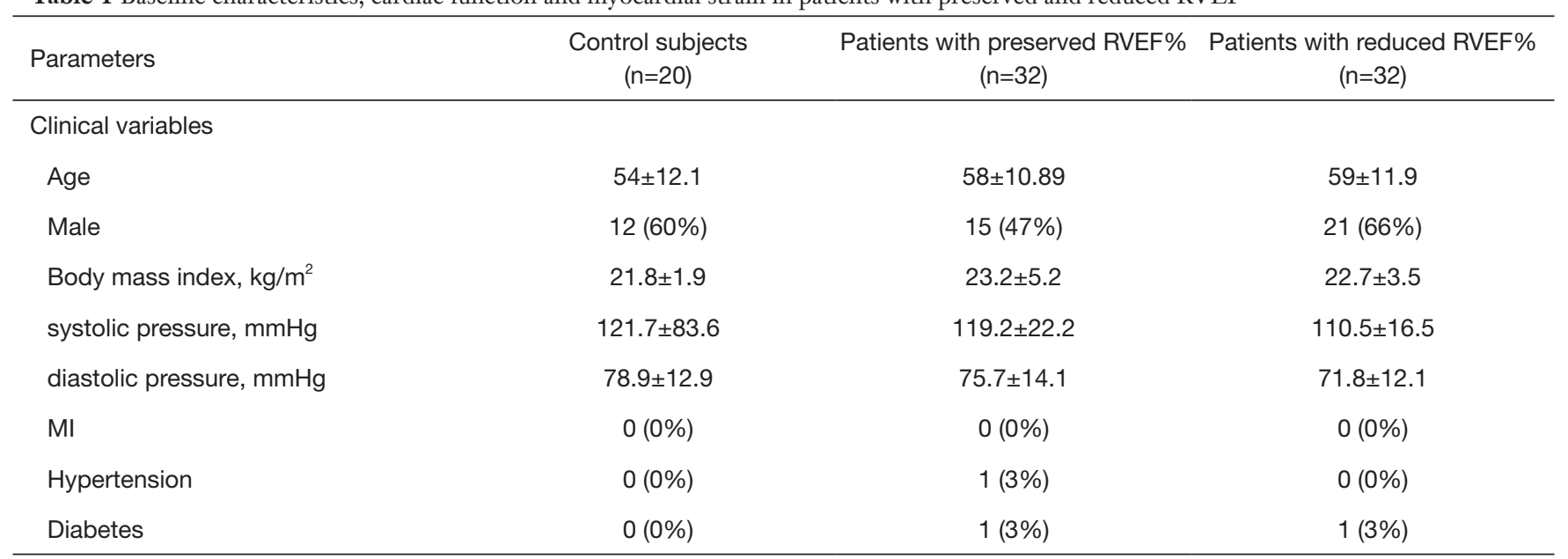

Table 1 (Continued) 
Table 1 (Continued)

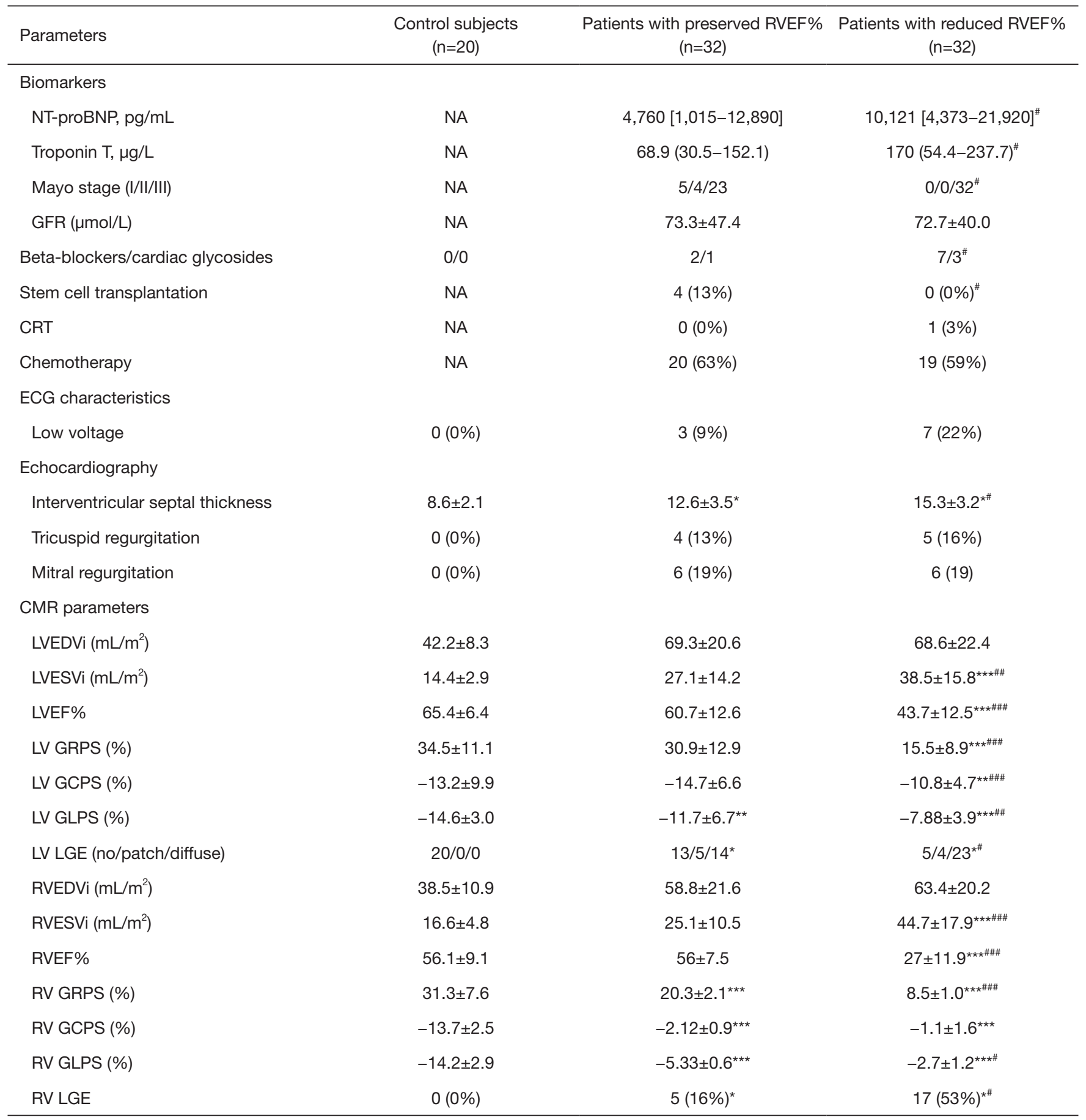

*, $\mathrm{P}<0.05 ;{ }^{* *}, \mathrm{P}<0.01 ;{ }^{* *}, \mathrm{P}<0.001$ versus control subjects. ${ }^{\#}, \mathrm{P}<0.05 ;{ }^{\#}, \mathrm{P}<0.01 ;{ }^{\# \#}, \mathrm{P}<0.001$ versus patients with preserved RVEF\%. NT-proBNP, N terminal pro B type natriuretic peptide; MI, myocardial infraction; CMR, cardiac magnetic resonance; EDVi, end diastolic volume-index; ESVi, end systolic volume-index; LVEF, left ventricular ejection fraction; RVEF, right ventricular ejection fraction; GCPS, global circumferential peak strain; GLPS, global longitudinal peak strain; GRPS, global radial peak strain; GFR, Glomerular filtration rate; CRT, cardiac resynchronization therapy; LGE, late gadolinium enhancement. 

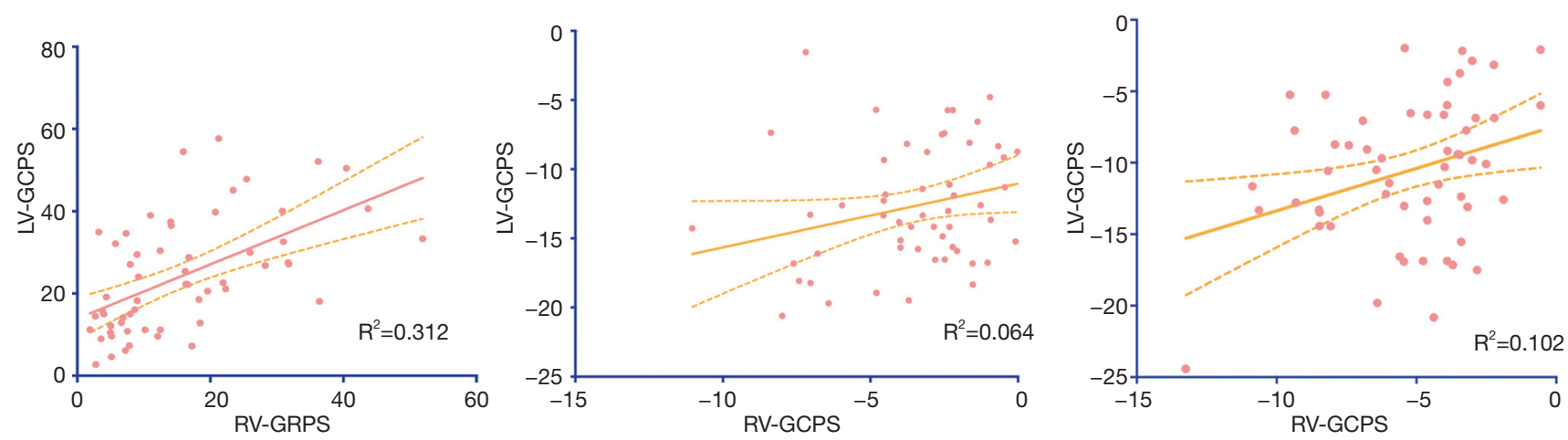

Figure 3 Pearson correlation analysis of GRPS, GCPS and GLPS between RV and LV. The Pearson correlation analysis showed that there were significant correlations between RV-GRPS and LV-GRPS ( $r=0.56, \mathrm{P}=0.001$ ), RV-GLPS and RV-LVGLPS ( $\mathrm{r}=0.32, \mathrm{P}=0.015$ ). The dotted line represents the $95 \%$ confidence interval.

Table 2 Comparison of clinical and RV strain in patients with or without CA

\begin{tabular}{|c|c|c|c|}
\hline RV strain & Control subjects $(n=20)$ & Patients without CA $(n=13)$ & Patients with CA $(n=51)$ \\
\hline RVESVi $\left(\mathrm{mL} / \mathrm{m}^{2}\right)$ & $27.9 \pm 8.7$ & $26.5 \pm 10.2$ & $37.3 \pm 18.6^{\star \#}$ \\
\hline RVEF\% & $56.1 \pm 9.1$ & $53 \pm 14.34$ & $41.5 \pm 17.3^{\star \star \star \# \# \#}$ \\
\hline RV GRPS (\%) & $31.3 \pm 7.6$ & $29.7 \pm 3.5^{\star \star \star}$ & $12.3 \pm 1.3^{\star \star \star \star \# \# ~}$ \\
\hline RV GLPS (\%) & $-14.24 \pm 2.9$ & $-5.7 \pm 2.1^{\star \star \star}$ & $-3.8 \pm 2.3^{\star \star \star \#}$ \\
\hline RV LGE & $0(0 \%)$ & $0(0 \%)$ & $22(43 \%)$ \\
\hline
\end{tabular}

*, $\mathrm{P}<0.05$; ${ }^{* *}, \mathrm{P}<0.001$ versus control subjects. " $\mathrm{P}<0.05$; \#\#\# $\mathrm{P}<0.001$ versus patients without $\mathrm{CA}$. EDVi, end diastolic volume-index; ESVi, end systolic volume-index; RVEF, right ventricular ejection fraction; GCPS, global circumferential peak strain; GLPS, global longitudinal peak strain; GRPS, global radial peak strain; late gadolinium enhancement.

Table 3 Comparison of biomarkers and RV strain in patients with or without RV LGE among CA patients

\begin{tabular}{|c|c|c|c|}
\hline RV strain & Control subjects $(n=20)$ & Patients without RV LGE $(n=22)$ & Patients with RV LGE $(n=29)$ \\
\hline RVESVi $\left(\mathrm{mL} / \mathrm{m}^{2}\right)$ & $27.9 \pm 8.7$ & $31.2 \pm 17.6$ & $46.2 \pm 17.3^{\star \star \star \#}$ \\
\hline RVEF\% & $56.1 \pm 9.1$ & $45.5 \pm 12.7^{\star \star}$ & $31.7 \pm 15.6^{\star \star \star \# \# \#}$ \\
\hline RV GRPS (\%) & $31.3 \pm 7.6$ & $13.8 \pm 9.6^{\star \star \star}$ & $7.1 \pm 4.3^{\star \star \star \# \#}$ \\
\hline RV GLPS (\%) & $-14.2 \pm 2.9$ & $-3.6 \pm 5.8^{\star \star \star}$ & $-3.9 \pm 3.8^{\star \star \star}$ \\
\hline
\end{tabular}

**, $\mathrm{P}<0.01$; ${ }^{* *}, \mathrm{P}<0.001$ versus control subjects., $\mathrm{P}<0.05$; ${ }^{\# \#, ~} \mathrm{P}<0.01$; \#\#\#, $\mathrm{P}<0.001$ versus patients without RV LGE. EDVi, end diastolic volume-index; ESVi, end systolic volume-index; RVEF, right ventricular ejection fraction; GCPS, global circumferential peak strain; GLPS, global longitudinal peak strain; GRPS, global radial peak strain; 
Table 4 Univariate models of clinical and CMR parameters predicting mortality in AL patients

\begin{tabular}{|c|c|c|}
\hline \multirow{2}{*}{ Parameters } & \multicolumn{2}{|c|}{ Univariate analysis } \\
\hline & $\mathrm{HR}(95 \% \mathrm{Cl})$ & $P$ value \\
\hline Age & $0.99(0.97-1.02)$ & 0.522 \\
\hline Gender & $0.89(0.48-1.65)$ & 0.711 \\
\hline BMI & $0.94(0.89-1.03)$ & 0.177 \\
\hline systolic pressure & $0.99(0.98-1.01)$ & 0.370 \\
\hline diastolic pressure & $0.98(0.95-1.01)$ & 0.096 \\
\hline Hypertension & $1.27(0.39-4.15)$ & 0.687 \\
\hline Diabetes & $0.73(0.26-2.07)$ & 0.543 \\
\hline Log NT-pro BNP & 2.75 (1.52-4.98) & $<0.001$ \\
\hline Log troponin $\mathrm{T}$ & 3.58 (1.82-7.04) & $<0.001$ \\
\hline Mayo stage & 3.39 (1.50-7.68) & 0.003 \\
\hline RVEF\% & $0.99(0.97-1.01)$ & 0.097 \\
\hline RV-LGE & $0.53(0.29-0.99)$ & 0.046 \\
\hline RV-GRPS & $0.92(0.88-0.96)$ & $<0.001$ \\
\hline RV-GCPS & $1.01(0.96-1.07)$ & 0.632 \\
\hline RV-GLPS & $1.12(1.03-1.22)$ & 0.006 \\
\hline LVEF\% & $0.97(0.95-0.99)$ & 0.001 \\
\hline LV-GRPS & $0.96(0.93-0.99)$ & 0.002 \\
\hline LV-GCPS & $1.14(1.06-1.22)$ & 0.001 \\
\hline LV-GLPS & $1.07(1.03-1.11)$ & 0.001 \\
\hline
\end{tabular}

For categorical data entered into the univariate and multivariate logistic regression models, indicator $=1$, as follows: gender, male $=1$, female $=0$; diabetes, positive $=1$, negative $=0$; hypertension, positive =1, negative =0; LGE, positive $=1$, negative $=0$; RVEF, GRPS, GLPS, GCPS increased by $1 \%$. LVEF, left ventricular ejection fraction; RVEF, right ventricular ejection fraction; GCPS, global circumferential peak strain; GLPS, global longitudinal peak strain; GRPS, global radial peak strain; late gadolinium enhancement.

\section{The prognostic value of $R V$ myocardial strain}

During a mean follow-up period of 15 months, there were $37(58 \%)$ deaths among all 64 included AL patients. Ten deaths occurred within 5 months of the AL amyloidosis diagnosis, 21 occurred within 10 months, 33 occurred within 15 months and 37 occurred within 20 months. Tables 4 and 5 reports the univariate and multivariate analyses of $\mathrm{AL}$ patients with various clinical and CMR parameters. In the univariate analysis, log NT-proBNP, log troponin
T, Mayo stage, RV-LGE, RV-GRPS, RV-GLPS, LVEF, LV-GRPS, LV-GCPS and LV-GLPS were significantly $(\mathrm{P}<0.05)$ related to survival. In the multivariate analysis of RV parameters and Mayo stage, only RV-GRPS (HR $=0.93$, 95\% CI, 0.88-0.98, $\mathrm{P}=0.007)$ and Mayo stage (HR =3.11, 95\% CI: $1.30-7.41, \mathrm{P}=0.01)$ remined strong predictors of mortality. In the multivariate analysis of RV and LV parameters, only RV-GRPS (HR $=0.92,95 \%$ CI: $0.87-0.97$, $\mathrm{P}=0.004)$ remined a strong predictor of mortality. As shown in Figure 4, patients with lower RV-GRPS had a significantly poorer prognosis than was found in those with higher RV-GRPS, based on the median CMR strain values (15.5\%) for GRPS (10 vs. 20 months, HR =0.93, 95\% CI: $0.87-0.98$, log-rank test $=9.712, \mathrm{P}=0.002)$.

\section{Intra-observer and inter-observer variability}

The intra-observer and inter-observer variability of all RV strain values obtained using CMR tissue tracking were calculated. Intra-observer and inter-observer reproducibility were excellent among RV GRPS and RV GLPS measurements, while the inter-observer of RV-GCPS was in general consistent. The ICCs of the results are summarized in Table 6. The ICCs of intra-observer variability for the measurements were 0.826-0.972; while the ICCs of interobserver variability for the measurements were $0.758-0.971$.

\section{Discussion}

The main findings of the present study indicate that RV strain derived from CMR tissue tracking may be vital for the early diagnosis of subclinical $\mathrm{RV}$ involvement in $\mathrm{AL}$ patients, especially in AL patients with preserved RVEF. More importantly, besides the traditional prognostic value of Mayo stage, we also found the RV GRPS and Mayo stage contributed significant prognostic information in AL patients after adjusting for $\mathrm{LV}$ parameters and traditional RV parameters, including RVEF and RV LGE.

In $\mathrm{AL}$ patients, $\mathrm{RV}$ heart failure often represents the late stage of the disease, in which a narrow therapeutic time window remains available. AL patients with impaired systolic function are considered inappropriate for autologous stem cell transplantation, which is generally accepted as a therapeutic approach to improve the survival rate and quality of life in $\mathrm{AL}$ amyloidosis $(26,27)$. Thus, early assessment of RV deformation plays a key role in therapeutic decision-making and prognosis evaluation in AL patients. As demonstrated in Cappelli's study, RV 
Table 5 Multivariate models of Mayo stage and CMR parameters predicting mortality in AL patients

\begin{tabular}{|c|c|c|c|c|c|c|}
\hline \multirow{2}{*}{ Parameters } & \multicolumn{3}{|c|}{ Multivariate models* } & \multicolumn{3}{|c|}{ Multivariate models* } \\
\hline & Wald Chi-square & $\mathrm{HR}(95 \% \mathrm{Cl})$ & $P$ value & Wald Chi-square & $\mathrm{HR}(95 \% \mathrm{Cl})$ & $P$ value \\
\hline Mayo stage & 6.58 & $3.11(1.30-7.41)$ & 0.010 & & & \\
\hline RV-GRPS & 7.34 & $0.93(0.88-0.98)$ & 0.007 & 8.255 & $0.92(0.87-0.97)$ & 0.004 \\
\hline RV-GLPS & 0.08 & $1.02(0.89-1.16)$ & 0.776 & & & \\
\hline LV-GRPS & & & & 0.871 & $1.04(0.96-1.12)$ & 0.351 \\
\hline LV-GCPS & & & & 1.087 & $1.11(0.91-1.34)$ & 0.297 \\
\hline LV-GLPS & & & & 1.687 & $1.04(0.98-1.11)$ & 0.194 \\
\hline
\end{tabular}

*, as limited number of cases included, this statistical method can not include more than five parameters. The left table included Mayo and right ventricular parameters, while the right table included left ventricular parameters and right ventricular parameter that has statistical significance. The two tables have the same header and statistical method. CMR, cardiac magnetic resonance; LVEF, left ventricular ejection fraction; GCPS, global circumferential peak strain; GLPS, global longitudinal peak strain; GRPS, global radial peak strain; late gadolinium enhancement.

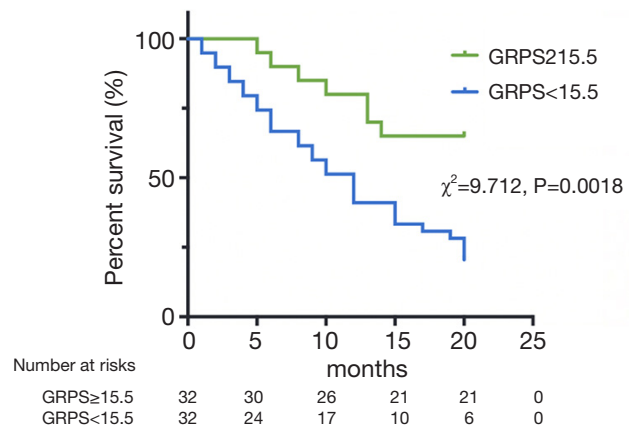

Figure 4 Prognostic value of RV GRPS for all-cause mortality in AL patients. Kaplan-Meier analysis revealed patients with lower RV-GRPS $<15.5$ had lower median survival time compared to patients with RVGRPS $\geq 15.5$ [12 vs. 20 months, (hazard ratio (HR) $=0.93$, 95\% CI: $0.87-$ 0.98, $\mathrm{P}=0.002]$. RV, right ventricular; GRPS, global radial peak strain.

Table 6 Intraclass correlation coefficient (ICC) analysis of GRPS, GCPS and GLPS

\begin{tabular}{lcc}
\hline Parameters & Cronbach's $\alpha$ & $95 \% \mathrm{Cl}$ \\
\hline GRPS & 0.972 & $0.938-0.988$ \\
Intra-observer & 0.971 & $0.936-0.978$ \\
Inter-observer & & \\
GCPS & 0.826 & $0.612-0.922$ \\
Intra-observer & 0.758 & $0.499-0.889$ \\
Inter-observer & & \\
GLPS & 0.92 & $0.822-0.964$ \\
Intra-observer & 0.853 & $0.672-0.934$ \\
Inter-observer &
\end{tabular}

GCPS, global circumferential peak strain; GLPS, global longitudinal peak strain; GRPS, global radial peak strain. longitudinal strain was significantly lower in AL patients with CA than in controls but remined normal in patients without CA (18). Accordingly, the present study also showed that patients with CA or RV LGE had lower RV strain than was found in patients without CA. Some studies have suggested that RV strain is correlated with LV filling pressure (28), and because of its anatomical position, the interventricular septum has been identified as a key element in ventricular interactions (29). In the correlation analysis performed in our study, there was a moderate correlation of GRPS and GLPS between LV and RV.

The prognosis of AL patients is often a paramount clinical concern. Some studies have demonstrated that NT-proBNP, troponin T, RV dysfunction and RV-LGE have prognostic value in $\mathrm{AL}$ patients (7,30-33). The prognostic value of CMR tissue tracking in AL patients has not yet been analysed. Moreover, whether the RV strain could provide information after adjusting for potent CMR or biomarkers remains uncertain. In our results, LVEF and LV strain parameters, Mayo stage, log NT-proBNP, log troponin T, RV-LGE, RV-GRPS and RV-GLPS were all risk factors for survival in univariate analysis. Nevertheless, only RV-GRPS and Mayo stage retained prognostic value for mortality in AL patients in the multivariate analysis after adjustment for other factors, demonstrating that RV-GRPS and Mayo stage are sensitive indicators of RV dysfunction in AL patients.

The CMR tissue tracking technique is an important modality that allows the assessment of regional myocardial function and the detection of any ventricular dysfunction in very early stages. Due to the accessibility of steady-state free recession (SSFP) cine images, CMR tissue tracking is the 
most commonly used sequence in clinical cardiac function assessment (34). Maceira et al. reported that the CMR-tissue tracking technique, when used for strain analysis, was a feasible and highly reproducible technique with an excellent intra-observer ICC (ICC >0.90) for strain (35). In our study, excellent intra-observer (ICC 0.92-0.97) and inter-observer (0.85-0.97) agreement was observed in RV-GRPS and RVGLPS. Therefore, RV deformation assessed by the CMR tissue tracking technique could provide substantial value in future clinical applications.

To our knowledge, this is the first investigation to test the usefulness of the CMR tissue tracking technique in evaluating RV deformation in AL patients. In this study, we analysed the feasibility and value of the CMR tissue tracking technique in obtaining early subclinical diagnoses and prognoses in AL patients.

Interestingly, unlike in the $\mathrm{LV}$, in which circumferential strain is more reproducible (36), in our study, we found that reproducibility measurements were moderate for RVGCPS. We speculate that this finding is related to the anatomical structure of the $\mathrm{RV}$, which is mainly composed of shallow myocardial layers that are arranged annularly and the deep myocardium, which is arranged longitudinally. This is a new finding with regard for the evaluation of RV deformation by $3 \mathrm{D}$ motion analysis but still require more research to verify.

There are some limitations to our study. First, the myocardium of the $\mathrm{RV}$ is too thin, and this makes it difficult to track the inner and outer myocardial membranes. However, because of the deposition of amyloidosis, AL patients have a greater RV myocardial thickness than is found in patients with other diseases. This makes it much easier to track the RV myocardial membranes in $\mathrm{AL}$ patients. Second, the follow-up time of 20 month may have been too short in the current study though previous studies have reported that clinical outcomes are poor in untreated $\mathrm{AL}$, in which the median survival is only 6 to 15 months (37). Finally, we did not assess T1 mapping or extracellular volume in the present study, which are well-known prognostic indicators $(38,39)$. However, as myocardial strain is an early marker of myocardial deformation, tissue tracking could detect RV deformation in the early stage of disease and easy to obtain.

\section{Conclusions}

Our findings demonstrate that the use of RV global strain evaluated by CMR tissue tracking in AL patients may be useful for the assessment of RV deformation. CMR RV strain tended to decrease in subclinical CA patients prior to RVEF reduction and provided valuable information for the early diagnosis of RV involvement. Moreover, RV-GRPS and Mayo stage served as a predictive factors for all-cause mortality in AL patients.

\section{Acknowledgments}

Funding: This study has received funding by the National Natural Science Foundation of China (No. 81771897), Program for New Century Excellent Talents in University (No. NCET-13-0386), Applied Basic Research Program of Science and Technology Commission Foundation of Sichuan Province (2017JY0027) of China and Program for Young Scholars and Innovative Research Team in Sichuan Province (2017TD0005) of China. 1.3.5 project for disciplines of excellence, West China Hospital, Sichuan University (ZYGD18013).

\section{Footnote}

Conflicts of Interest: All authors have completed the ICMJE uniform disclosure form (available at http://dx.doi. org/10.21037/cdt.2020.01.03). The authors have no conflicts of interest to declare.

Ethical Statement: All authors are accountable for all aspects of the work in ensuring that questions related to the accuracy or integrity of any part of the work are appropriately investigated and resolved. This study was approved by our institutional review board (No. 2019-756).

Open Access Statement: This is an Open Access article distributed in accordance with the Creative Commons Attribution-NonCommercial-NoDerivs 4.0 International License (CC BY-NC-ND 4.0), which permits the noncommercial replication and distribution of the article with the strict proviso that no changes or edits are made and the original work is properly cited (including links to both the formal publication through the relevant DOI and the license). See: https://creativecommons.org/licenses/by-nc-nd/4.0/.

\section{References}

1. Milani P, Merlini G, Palladini G. Light Chain Amyloidosis. Mediterr J Hematol Infect Dis 2018;10:e2018022.

2. Palladini G, Merlini G. What is new in diagnosis 
and management of light chain amyloidosis. Blood 2016;128:159-68.

3. Wechalekar AD, Gillmore JD, Hawkins PN. Systemic amyloidosis. Lancet 2016;387:2641-54.

4. Heiberg J, Ringgaard S, Schmidt MR, et al. Structural and functional alterations of the right ventricle are common in adults operated for ventricular septal defect as toddlers. Eur Heart J Cardiovasc Imaging 2015;16:483-9.

5. Schmidt R, Orwat S, Kempny A, et al. Value of speckletracking echocardiography and MRI-based feature tracking analysis in adult patients after Fontan-type palliation. Congenit Heart Dis 2014;9:397-406.

6. Kempny A, Fernández-Jiménez R, Orwat S, et al. Quantification of biventricular myocardial function using cardiac magnetic resonance feature tracking, endocardial border delineation and echocardiographic speckle tracking in patients with repaired tetralogy of Fallot and healthy controls. J Cardiovasc Magn Reson 2012;14:32.

7. van Kessel M, Seaton D, Chan J, et al. Prognostic value of right ventricular free wall strain in pulmonary hypertension patients with pseudo-normalized tricuspid annular plane systolic excursion values. Int J Cardiovasc Imaging 2016;32:905-12.

8. Venner C, Selton-Suty C, Huttin O, et al. Right ventricular dysfunction in patients with idiopathic dilated cardiomyopathy: Prognostic value and predictive factors. Arch Cardiovasc Dis 2016;109:231-41.

9. Claus P, Omar AMS, Pedrizzetti G, et al. Tissue Tracking Technology for Assessing Cardiac Mechanics: Principles, Normal Values, and Clinical Applications. JACC Cardiovasc Imaging 2015;8:1444-60.

10. Gavara J, Rodriguez-Palomares JF, Valente F, et al. Prognostic Value of Strain by Tissue Tracking Cardiac Magnetic Resonance After ST-Segment Elevation Myocardial Infarction. JACC Cardiovasc Imaging 2018;11:1448-57.

11. Liu X, Zhang Q, Yang ZG, et al. Assessment of left ventricular deformation in patients with Ebstein's anomaly by cardiac magnetic resonance tissue tracking. Eur J Radiol 2017;89:20.

12. Zghaib T, Ghasabeh MA, Assis FR, et al. Regional Strain by Cardiac Magnetic Resonance Imaging Improves Detection of Right Ventricular Scar Compared With Late Gadolinium Enhancement on a Multimodality Scar Evaluation in Patients With Arrhythmogenic Right Ventricular Cardiomyopathy. Circ Cardiovasc Imaging 2018;11:e007546.

13. Illman JE, Arunachalam SP, Arani A, et al. MRI feature tracking strain is prognostic for all-cause mortality in AL amyloidosis. Amyloid 2018;25:101-8.

14. Buss SJ, Breuninger K, Lehrke S, et al. Assessment of myocardial deformation with cardiac magnetic resonance strain imaging improves risk stratification in patients with dilated cardiomyopathy. Eur Heart J Cardiovasc Imaging 2015;16:307-15.

15. Li R, Yang ZG, Xu HY, et al. Myocardial Deformation in Cardiac Amyloid Light-chain Amyloidosis: Assessed with 3T Cardiovascular Magnetic Resonance Feature Tracking. Sci Rep 2017;7:3794.

16. Tadic M. Multimodality Evaluation of the Right Ventricle: An Updated Review. Clin Cardiol 2015;38:770-6.

17. Champion HC, Michelakis ED, Hassoun PM. Comprehensive invasive and noninvasive approach to the right ventricle-pulmonary circulation unit: state of the art and clinical and research implications. Circulation 2009;120:992-1007.

18. Cappelli F, Porciani MC, Bergesio F, et al. Right ventricular function in AL amyloidosis: characteristics and prognostic implication. Eur Heart J Cardiovasc Imaging 2012;13:416-22.

19. Cantinotti M, Bell A, Razavi R. Role of magnetic resonance imaging in different ways of presentation of Ebstein's anomaly. J Cardiovasc Med (Hagerstown) 2008;9:628-30.

20. Alfakih K, Plein S, Bloomer T, et al. Comparison of right ventricular volume measurements between axial and short axis orientation using steady-state free precession magnetic resonance imaging. J Magn Reson Imaging 2003;18:25-32.

21. Wan K, Sun J, Yang D, et al. Left Ventricular Myocardial Deformation on Cine MR Images: Relationship to Severity of Disease and Prognosis in Light-Chain Amyloidosis. Radiology 2018;288:73-80.

22. Bhatti S, Vallurupalli S, Ambach S, et al. Myocardial strain pattern in patients with cardiac amyloidosis secondary to multiple myeloma: a cardiac MRI feature tracking study. Int J Cardiovasc Imaging 2018;34:27-33.

23. Jaccard A, Moreau P, Leblond V, et al. High-dose melphalan versus melphalan plus dexamethasone for AL amyloidosis. N Engl J Med 2007;357:1083-93.

24. Gertz MA, Comenzo R, Falk RH, et al. Definition of organ involvement and treatment response in immunoglobulin light chain amyloidosis (AL): a consensus opinion from the 10th International Symposium on Amyloid and Amyloidosis, Tours, France, 18-22 April 2004. Am J Hematol 2005;79:319-28.

25. Aschauer S, Kammerlander AA, Zotter-Tufaro C, et al. 
The right heart in heart failure with preserved ejection fraction: insights from cardiac magnetic resonance imaging and invasive haemodynamics. Eur J Heart Fail 2016;18:71-80.

26. Skinner M, Sanchorawala V, Seldin DC, et al. High-dose melphalan and autologous stem-cell transplantation in patients with AL amyloidosis: an 8-year study. Ann Intern Med 2004;140:85-93.

27. Cibeira MT, Sanchorawala V, Seldin DC, et al. Outcome of AL amyloidosis after high-dose melphalan and autologous stem cell transplantation: long-term results in a series of 421 patients. Blood 2011;118, 4346-52.

28. Motoji Y, Tanaka H, Fukuda Y, et al. Interdependence of right ventricular systolic function and left ventricular filling and its association with outcome for patients with pulmonary hypertension. Int J Cardiovasc Imaging 2015;31:691-8.

29. Buckberg GD, RESTORE Group. The ventricular septum: the lion of right ventricular function, and its impact on right ventricular restoration. Eur J Cardiothorac Surg 2006;29:S272-8.

30. Prota C, Di Salvo G, Sabatino J, et al. Prognostic value of echocardiographic parameters in pediatric patients with Ebstein's anomaly. Int J Cardiol 2019;278:76-83.

31. Wan K, Sun J, Han Y, et al. Right ventricular involvement evaluated by cardiac magnetic resonance imaging predicts mortality in patients with light chain amyloidosis. Heart Vessels 2018;33:170-9.

32. Ghio S, Perlini S, Palladini G, et al. Importance of the echocardiographic evaluation of right ventricular function in patients with AL amyloidosis. Eur J Heart Fail
2007;9:808-13.

33. Palladini G, Campana C, Klersy C, et al. Serum N-terminal pro-brain natriuretic peptide is a sensitive marker of myocardial dysfunction in AL amyloidosis. Circulation 2003;107:2440-5.

34. Almutairi HM, Boubertakh R, Miquel ME, et al. Myocardial deformation assessment using cardiovascular magnetic resonance-feature tracking technique. Br J Radiol 2017;90:20170072.

35. Maceira AM, Tuset-Sanchis L, López-Garrido M, et al. Feasibility and reproducibility of feature-tracking-based strain and strain rate measures of the left ventricle in different diseases and genders. J Magn Reson Imaging 2018;47:1415-25.

36. Schuster A, Paul M, Bettencourt N, et al. Cardiovascular magnetic resonance myocardial feature tracking for quantitative viability assessment in ischemic cardiomyopathy. Int J Cardiol 2013;166:413-20.

37. Kyle RA, Gertz MA. Primary systemic amyloidosis: clinical and laboratory features in 474 cases. Semin Hematol 1995;32:45-59.

38. Lin L, Li X, Feng J, et al. The prognostic value of T1 mapping and late gadolinium enhancement cardiovascular magnetic resonance imaging in patients with light chain amyloidosis. J Cardiovasc Magn Reson 2018;20:2.

39. Banypersad SM, Sado DM, Flett AS, et al. Quantification of Myocardial Extracellular Volume Fraction in Systemic AL Amyloidosis: An Equilibrium Contrast Cardiovascular Magnetic Resonance Study. Circ Cardiovasc Imaging 2013;6:34-9.
Cite this article as: Liu H, Fu H, Guo YK, Yang ZG, Xu HY, Shuai X, Xu R, Li ZL, Xia CC, He Y, Zhou XY. The prognostic value of right ventricular deformation derived from cardiac magnetic resonance tissue tracking for all-cause mortality in light-chain amyloidosis patients. Cardiovasc Diagn Ther 2020;10(2):161-172. doi: 10.21037/cdt.2020.01.03 